\section{Increasing the utility of negative instances in conjunctive concept identification}

\author{
JOSEPH F, BRINLEY and ROBERT J. \\ SARDELLO, 1 St. Louis University, \\ St. Louis, Mo. 63103
}

Two groups of $S$ s performed concept identifications under different conditions. Problems in which information was conveyed exclusively in negative instances were significantly more difficult than other problems in one condition but not in the other. Results are interpreted in relation to differences in strategy and memory requirements in the two conditions.

Following Smoke (1932), several studies (e.g., Hovland \& Weiss, 1953; Freibergs \& Tulving, 1961; Haygood \& Devine, 1967) have probed questions related to the difficulty of using negative information in solving concept-identification problems. The point of departure for the experiment reported here was found in studies by Huttenlocher $(1962,1964)$.

Huttenlocher used problems similar to the A problems diagrammed in Fig. 1. Information about the "correct" conjunction of color and shape attributes was conveyed in two successive sets of instances, with each set designated either positive $(+)$ or negative (-). All combinations of sequences of sets of instances $(++,+-,-+,--)$ were employed. The -- problems proved most difficult. This finding was highly reliable, and other studies (e.g., Hovland \& Weiss, 1953) have also found it more difficult to use negative information when only negative instances are presented than when negative instances are intermixed with positive instances.

The experiment reported here was an associated with the use of -- sequences in a problem situation of the type employed by Huttenlocher. The B problems in Fig. 1 differed from the A problems in only two ways: (1) They provided explicit information about the entire set of possible solutions so that there was no need to generate this in order to solve -problems; (2) they eliminated the need for $S$ to remember information from prior presentations in order to use it in relation to information presented subsequently. B problems thus made it unnecessary to discover and to use the formal or hypothetico-deductive strategies involving higher memory loads that were indispensable for solving -- sequences in the $A$ problems. attempt to reduce or to eliminate difficulty
The B problems were presented in three slides. The first slide presented the entire set of possibly relevant concepts. The second and third slides were identical with the first two slides of the A problems, except that each also re-presented the information presented in the one or two slides preceding it.

\section{PROCEDURE}

Slides were presented with a Carousel projector. The presentation interval was $10 \mathrm{sec}$, with no delay between slides except for a $20-\mathrm{sec}$ interproblem interval. The 24 problems in each condition were presented in quasirandom order. After a brief rest, the same problems were then presented in

\section{MATERIALS}

Two sets of problems were constructed. Each set had 24 problems: six problems for each of the possible sequences,,,+++--+ , - - Two of the six problems for each sequence were constructed with two different shapes (as diagrammed), two others involved three different shapes, while two problems involved four different shapes. Since the correct solution in each problem was one of the shapes presented and was either black or white in color, this variation in the number of shapes varied the number of possibly relevant concepts from four to six to eight, a variation referred to as task complexity.

The A problems were presented in two slides. The first slide presented two, three, or four shapes, followed by either a + or a - sign, depending on whether or not the set included the "correct" instance. The first slide eliminated half the alternatives, and the second eliminated the uncertainty remaining. a different order. Ss were required to draw their answers in a test booklet.

Each experimental group was tested in a single session. The instructions were as follows:

"I am going to show you some pictures of geometric figures. These figures can either be white or they can be black. On the right-hand side of each picture you will see either $a+$ sign or a - sign. Your job is to figure out which one geometric figure in which color makes the sign on the right be plus. Let us do an example problem. Here is a black circle. On the right you see that there is a + sign. This means that, in this problem, the black circle made the sign be plus. On Page 1 of your booklet, draw a circle and fill it in. That is the way you will indicate your answer. Draw the figure that makes the sign on the right be plus.

"Here is another example. Here is a black $X$. On the right, there is a - sign. This means that the $X$ had to be white to

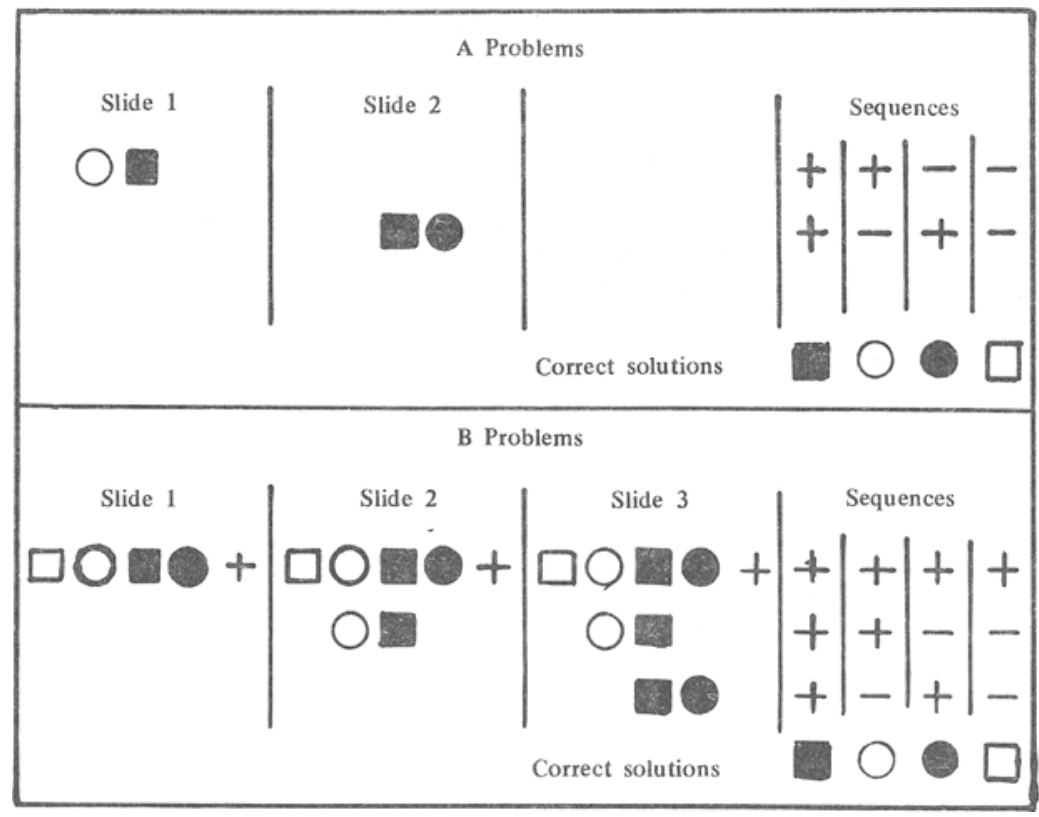

Fig. 1. Schematic for each type of problem 
make the sign on the right be plus. On Page 2, draw an $X$ and don't fill it in. Now, in the problems you will do, there will be more than one geometric figure, and you will have to figure out which one figure in which color makes the sign be plus. On each problem, you will be given enough information to solve the problem without guessing. Draw your answer when I say, 'Draw your answer,' and turn the page when I say, "Turn the page." "

The initial problem for both groups involved a -+ sequence, with two figures. This problem was presented three times in succession while Es checked to see whether or not Ss were following instructions in an appropriate fashion. The correct solution was not revealed.

\section{RESULTS}

Table 1 provides the main findings. Analysis of mean number correct on the A problems reveals a rank ordering of problem difficulty in relation to sequencing of sets of instances identical with that obtained by Huttenlocher. ANOVA found this main effect significant $(\mathrm{F}=16.51, \mathrm{df}=1 / 11, \mathrm{p}<.01)$. The -sequences were most difficult, and the Dunnett test for comparing each one of a number of means with a standard or control (Edwards, 1968) revealed significant differences between the -mean and each of the three other means ( $p<.01$ for each comparison).

Analysis did not reveal any relationship between sequencing and problem difficulty for the B problems $(F<1)$. There was no main effect associated with problem complexity for either A problems $(F<1)$ or $\mathrm{B}$ problems $(\mathrm{F}=1.45)$.

\section{DISCUSSION}

With A problems, this experiment found, as others have, that it was more difficult to identify conjunctive concepts when information was conveyed exclusively in negative instances than when only positive instances or when mixed series of negative and positive instances were employed. With B problems, there
Table 1

Means and SDs for Different Sequences of $A$ and B Problems

\begin{tabular}{ll|r|r|r|r}
\hline Sequence & & \multicolumn{1}{c}{-+} & \multicolumn{1}{c}{++} & \multicolumn{1}{c}{+} & \multicolumn{1}{c}{-} \\
\hline \multirow{4}{*}{ A Problems } & Mean & 8.92 & 7.42 & 5.58 & 1.08 \\
& SD & 2.32 & 4.92 & 4.17 & 2.14 \\
B Problems & Mean & 10.67 & 10.42 & 11.00 & 10.25 \\
& SD & 1.50 & 1.26 & 1.35 & 2.45 \\
\hline
\end{tabular}

was no evidence for any increased difficulty of - - sequences.

It seems clear that this outcome is related to the fact that conditions in the B problems eliminated the need for developing and using the kind of strategy indispensable for solving - - sequences in A problems. The - - sequences served to eliminate all but one of the possible solutions that was designated correct. Consequently, it was necessary for $S$ to have information about the complete set of possible concepts in order to solve any -problem. Information about all possible concepts was given directly to $S$ in the B problems. In A problems, $S$ had to develop and use the sort of hypothetico-deductive strategy that would generate this information.

What is not clear from the results is whether the greater difficulty of -sequences in A problems was associated with failure to develop an appropriate strategy or with difficulty in using one. Conditions did not favor the development of an appropriate strategy. So long as one instance was positive, $\mathrm{S}$ could solve a problem by simple induction: With +t sequences, the correct concept appeared in both sets of instances; with +- or -t sequences, the correct concept was present in the positive set but not in the negative one. Only the - sequences required the more indirect, and presumably less obvious or less available, "hypothetico-deductive approach. Consequently, $75 \%$ of the problems could be solved without employing the type of strategy needed for the A - sequences. Ss could have been set for inappropriate strategies, even though they might have employed appropriate ones under more favorable circumstances.

It is also possible that the greater difficulty of -- sequences in A problems was associated with difficulty in using an appropriate strategy rather than with inability or disinclination to use one. This would be true if, as Huttenlocher (1962, 1964) seems to assume, Ss tend to employ formal or hypothetico-deductive reasoning in solving problems regardless of sequencing of instances. The factor here may be memory load. Previous studies, e.g., Hovland \& Weiss (1953), have reported that reducing memory load by simultaneous presentation of instances diminishes the greater difficulty of processing information conveyed exclusively in negative instances. Since B problems not only provided the set of possible solutions but also reduced memory load by simultaneous presentation of instances, further work is required to decide whether one or both of the interpretations are correct.

\section{REFERENCES}

EDWARDS, A. L. Experimental design in psychological research. (2nd. ed.) New York: Holt, Rinehart \& Winston, 1968.

FREIBERGS, V., \& TULVING, E. The effect of practice on utilization of information from positive and negative instances in concept identification. Canadian Journal of Psychology, 1961, 15, 101-106.

HAYGOOD, R. C., \& DEVINE, J. V. Effects of composition of the positive category on concept learning. Journal of Experimental Psychology, 1967, 74, 230-235.

HOVLAND, C. I., \& WEISS, W. Transmission of information concerning concepts through positive and negative instances. Journal of Experimental Psychology, 1953, 45, 175-182.

HUTTENLOCHER, J. Some effects of negative instances on the formation of simple concepts. Psychological Reports, 1962, 11, 35-42.

HUTTENLOCHER, J. Development of formal reasoning on concept formation problems. Child Development, 1964, 35, 1233-1242.

SMOKE, R. L. An objective study of concept formation. Psychological Monographs, 1932, 42(4, Whole No, 191). NOTE

1. The second author is now at Duquesne University. 